\title{
Efeito de adubos foliares aplicados em mistura com fungicidas na cultura do amendoim
}

Submetido - 31 jul. $2020 \quad$ Aprovado - 05 set. $2020 \quad$ Publicado - 14 out. 2020
dol http://dx.doi.org/10.17648/sas.v1i1.75

Engenheiro Agrônomo - CENA/USP - e-mail: tulio.stelluti@hotmail.com.

Tulio Stelluti iD

Eng ${ }^{\underline{a}}$. Agrônoma Drª - APTA, e-mail: maria.soares@sp.gov.br.

Maria Beatriz Bernardes Soares ${ }^{(D)}$ Thaian Neves dos Santos ${ }^{D}$ Engenheiro Agrônomo-APTA/FUNDAG, e-mail: thaiansantos@hotmail.com.

Everton Luis Finoto

Engenheiro Agrônomo Dr-APTA Polo Regional Centro Norte, e-mail: evertonfinoto@apta.sp.gov.br.

Engenheiro Agrônomo Dr - Omnia Brasil, e-mail: eli.oliveira@omniabrasil.com.br.

\section{RESUMO}

O uso de substâncias húmicas além de promover uma maior disponibilidade de nutrientes as plantas, pelo aumento da capacidade de troca catiônica (CTC) no solo, promove também uma melhoria na qualidade do solo e a interação solo-planta. $O$ objetivo deste trabalho foi avaliar a compatibilidade da adubação foliar com a aplicação de fungicidas na cultura do amendoim cultivar IAC 503. Foram aplicadas aos 35, 100, 121, 133 e 151 dias após o plantio. As doses dos produtos (Fungicidas e adubos foliares), foram seguidas as recomendações dos fabricantes. Fungicidas usados foram o Bravonil Ultrex (2 I. ha-1), o Engeo PlenoTM S (0,2 L.ha $\left.{ }^{-1}\right)$, o Pirate (1,2 L.ha'), o Opera (0,6 L.ha') e o Fastac Duo (0,2 L.ha-1). Os adubos foliares foram 0 Rhizovator (3 L. ha-1) e o SeedBoost $\left(0,5 \mathrm{~L}^{\left.-h^{-1}\right)}\right.$. Os tratamentos que receberam maiores números de aplicações foram os mais responsivos quanto ao aumento de produtividade com ganhos de 35,78 e 35,68\%, sendo T4 e T5, com 4 e 5 aplicações respectivamente. Os tratamentos T2 e T6, receberam apenas uma aplicação de substâncias húmicas, entretanto houve um incremento na produtividade de em 9,35 e 14\% quando comparado com a testemunha (T1). Portanto, uso de substâncias húmicas não apresenta problemas na compatibilidade com fungicidas em mistura no tanque, além de promover ganhos na produtividade de vagens.

Palavras-chave: Substâncias húmicas; Condicionadores de solo; Ácidos orgânicos.

\section{Effect of foliarfertilizers applied in mixture with fungicides in peanut crop}

\section{ABSTRACT}

The use of humic substances in addition to promoting greater availability of nutrients to plants, by increasing the cation exchange capacity (CTC) in the soil, also promotes an improvement in soil quality and soil-plant interaction. The objective of this work was to evaluate the compatibility of foliar fertilization with the application of fungicides in the culture of the peanut cultivar IAC 503. They were applied at 35, 100, 121, 133 and 151 days after planting. The doses of the products (Fungicides and foliar fertilizers) were followed by the manufacturers' recommendations. Fungicides used were Bravonil Ultrex (2 I.ha-1), Engeo PlenoTM S (0.2 L.ha-1), Pirate (1.2 L.ha $\left.{ }^{-1}\right)$, Opera (0.6 L .ha $\left.{ }^{-1}\right)$ and Fastac Duo (0.2 L.ha $\left.{ }^{-1}\right)$. Foliar fertilizers were Rhizovator (3 L.ha-1) and SeedBoost $\left(0.5\right.$ L.ha $\left.^{-1}\right)$. The treatments that received the highest number of applications were the most responsive to the increase in productivity with gains of 35.78 and $35.68 \%$, being T4 and T5, with 4 and 5 applications respectively. The T2 and T6 treatments received only one application of humic substances, however there was an increase 
in productivity of 9.35 and $14 \%$ when compared to the control (T1). Therefore, the use of humic substances does not present problems in the compatibility with fungicides mixed in the tank, besides promoting gains in the productivity of pods.

Keywords: Humic substances; Soil conditioner; Organic acids.

\title{
Efecto de los fertilizantes foliares aplicados en mezcla com fungicidas en el cultivo de maní.
}

\begin{abstract}
RESUMEN
El uso de sustancias húmicas, además de promover una mayor disponibilidad de nutrientes para las plantas, al incrementar la capacidad de intercambio catiónico (CTC) en el suelo, también promueve una mejora en la calidad del suelo y la interacción suelo-planta. El objetivo de este trabajo fue evaluar la compatibilidad de la fertilización foliar con la aplicación de fungicidas en el cultivo del maní cultivar IAC 503. Se aplicaron a los 35, 100, 121, 133 y 151 días después de la siembra. Las dosis de los productos (Fungicidas y fertilizantes foliares) fueron seguidas por las recomendaciones de los fabricantes. Los fungicidas utilizados fueron Bravonil Ultrex (2 I.ha-1), Engeo PlenoTM S (0,2 L.ha-1), Pirate (1,2 L.ha-1), Opera $(0,6$ L .ha-1) y Fastac Duo $\left(0,2\right.$ L.ha $\left.^{-1}\right)$. Los fertilizantes foliares fueron Rhizovator (3 L.ha-1) y SeedBoost $\left(0,5\right.$ L.ha $\left.^{-1}\right)$. Los tratamientos que recibieron mayor número de aplicaciones fueron los que más respondieron al incremento de productividad con ganancias de 35,78 y 35,68\%, siendo T4 y T5, con 4 y 5 aplicaciones respectivamente. Los tratamientos T2 y T6 recibieron solo una aplicación de sustancias húmicas, sin embargo hubo un aumento de productividad de 9.35 y $14 \%$ en comparación con el control (T1). Por tanto, el uso de sustancias húmicas no presenta problemas en la compatibilidad con fungicidas mezclados en el tanque, además de promover ganancias en la productividad de las vainas.
\end{abstract}

Palabras clave: Ácidos húmicos; Acondicionador de suelos; Ácidos orgânicos.

\section{Introdução}

O Estado de São Paulo é o maior produtor de amendoim, sendo as regiões localizadas nas chamadas Alta Mogiana, Alta Paulista (Marília e se expandindo para as regiões norte e noroeste as mais expressivas). De acordo com a Conab (2020), estimou um aumento de área cultivada de $9,33 \%, 17,3 \%$ a mais de produtividade $(\mathrm{kg} / \mathrm{ha})$ e $28,2 \%$ a mais na produção quando comparada com a safra passada. Suassuna (2008) e Ferreira (2014), relatam que a cultura do amendoim é acometida por doenças fungicas em todo seu ciclo, principalmente nas áreas foliares com predominâncias as cercosporioses.

A cultura do amendoim requer, em sua zona de desenvolvimento dos frutos, uma alta necessidade Cálcio, este é o terceiro nutriente mais exigido e $\mathrm{N}$ e $\mathrm{K}$ requeridos em maior quantidade (CRUSCIOL e SORATTO, 2007; FOLONI et al, 2016). A matéria orgânica do solo é dividida em: matéria orgânica viva e não vivente. Esta contribui em média com $98 \%$ do $C$ em forma orgânica, carbono orgânico total COT. O solo é um compartimento 
terrestre que se subdivide em: fração mineral e orgânica (SILVA, 2007). Esta corresponde em média $5 \%$ do teor no solo, dentro dessa fração temos a chamada fração estável da matéria orgânica do solo (MOS), sendo representado pelo húmus, fração mais estabilizada da matéria orgânica (MO), moléculas complexas de difícil acesso pelos microrganismos, sendo um compartimento que engloba as denominadas substâncias húmicas (SILVA, 2007).

Nesse contexto, com o advento de novas tecnologias e cultivares com altos tetos produtivos, há no mercado novas técnicas de manejo de adubação para incremento de produtividade. MAPA (2020), traz os chamados bioinsumos, estes recomendados para manutenção ou incremento da capacidade do solo em sustentar o crescimento e a produtividade das plantas. Sendo assim, há hoje no mercado produtos que possuem em sua composição elevados teores de compostos húmicos que irão auxiliar na manutenção da microbiota do solo, elevação de cátions de caráter básicos como, por exemplo, $\mathrm{Ca}^{2+}, \mathrm{K}^{+}$e $\mathrm{Mg}^{2+}$ e na disponibilidade de P, competindo pelos sítios de adsorção, liberando-o na solução (SILVA, 2007).

O presente trabalho teve por objetivo avaliar a compatibilidade de adubos foliares em mistura com fungicidas e o efeito dos tratamentos na produtividade do amendoim.

\section{Material e métodos}

O experimento foi conduzido na Agência Paulista de Tecnologia dos Agronegócios (APTA) - Polo Centro Norte, pertencente à Secretaria da Agricultura e Abastecimento do Estado de São Paulo, localizado no município de Pindorama, SP. A área experimental tem solo caracterizado como ARGISSOLO Eutrófico vermelho-amarelo, considerado profundo, com horizonte A arenoso e horizonte B textural com alta fertilidade e topografia plana. Conforme classificação de Koppen, o clima enquadra-se no tipo Aw, definido como tropical úmido com estação chuvosa no verão e seca no inverno. 
Utilizou-se delineamento em blocos casualizados (DBC), com quatro repetições e os tratamentos estão expostos na Tabela 1. A cultivar utilizada foi a IAC 503. As unidades experimentais foram constituídas de quatro linhas de cinco metros de comprimento, sendo a área útil as duas linhas centrais, espaçadas a 0,90 metros e a densidade de semeadura foi de 20 sementes por metro.

As doses dos produtos (Fungicidas e adubos foliares), foram seguidas as recomendações dos fabricantes. Fungicidas usados foram o Bravonil Ultrex (2 I.ha $\left.{ }^{-1}\right)$, o Engeo Pleno ${ }^{\text {TM }}$ S $\left(0,2\right.$ L.ha $\left.^{-1}\right)$, o Pirate (1,2 L.ha $\left.{ }^{-1}\right)$, o Opera (0,6 L.ha-1) e o Fastac Duo (0,2 L.ha- $\left.{ }^{-1}\right)$. Os adubos foliares foram o Rhizovator (3 L.ha-1) e o SeedBoost $\left(0,5\right.$ L.ha $\left.^{-1}\right)$.

As aplicações foram realizadas aos 35, 121, 133 e 149 dias após o plantio (DAP), na ocasião da operação de aplicação do fungicida e/ou inseticida. As aplicações dos produtos foram realizadas utilizando pulverizador terrestre na vazão de 300 L.ha $^{-1}$, equipados com pontas de pulverização TTJ60 - $11003110^{\circ}$ duplo leque espaçadas a 0,5 m.

Tabela 1. Tratamentos utilizados e época de aplicação em dias após do plantio (DAP). Pindorama (SP), 2020.

\begin{tabular}{cl}
\hline Tratamentos & \multicolumn{1}{c}{ ESPECIFICAÇÃO } \\
\hline T1 & Cultivo padrão regional \\
T2 & Rhizovator 3,0 L/ha aos 35 DAP \\
T3 & Seedboost 0,5 L/ha aos 121 DAP \\
T4 & Rhizovator 3,0 L/ha aos 100, 121, 133 e 150 \\
& DAP \\
T5 & T2 + T4 \\
T6 & Seedboost 1,0 L/ha aos 50 DAP \\
T7 & T2 + T3 \\
\end{tabular}

A avaliação da sintomatologia das doenças foi realizada momentos antes da aplicação dos fungicidas, nas linhas centrais da unidade experimental, através de uma escala diagramática com notas de sintomas visuais variando de 1 a 9 . Esta escala considera a quantidade de mancha 
preta por folha, o número de folhas com lesões e a desfolha ao longo de um dos ramos primários da planta (SUBRAHMANYAM et al., 1982).

Ao final do ciclo foi realizada a colheita das unidades experimentais nas duas linhas centrais, considerando $9,0 \mathrm{~m}^{2}$ para obtenção da produtividade em $\mathrm{kg} \mathrm{ha}^{-1}$.

Os dados foram submetidos à análise de variância pelo teste $F$, para efeito de significância dos tratamentos e as médias comparadas pelo teste de Tukey a $5 \%$ de probabilidade.

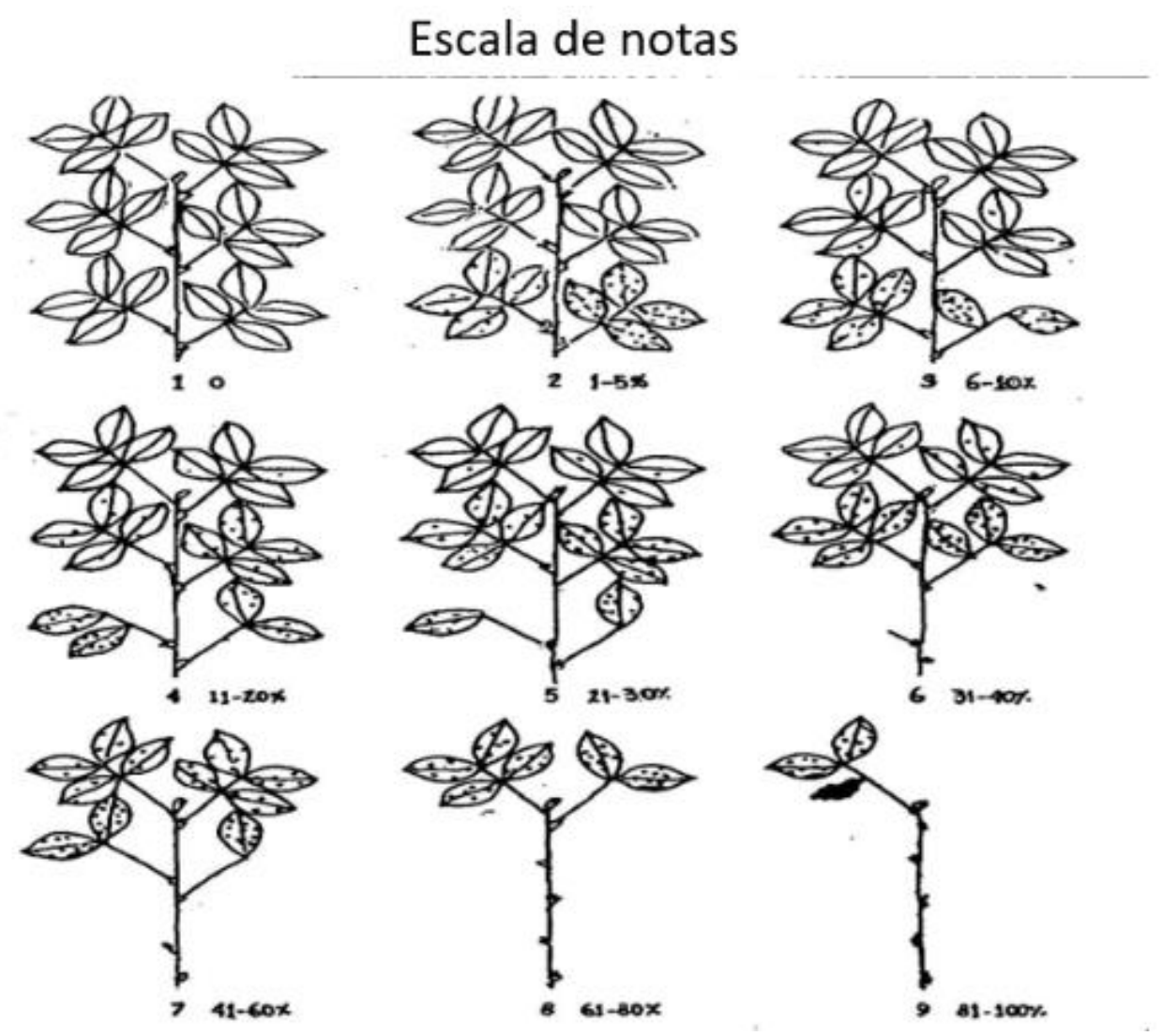

Figura 1. Escala de notas para doenças na cultura do amendoim. Pindorama, 2020.

\section{Resultados e discussão}

A análise de variância (ANOVA) demonstrou não haver influência significativa entre os tratamentos com uso de adubo foliar juntamente com 0 fungicida para o controle e períodos de avaliação dos sintomas visuais de mancha preta do amendoim (Tabela 2). 
Tabela 2. Notas de sintomas de mancha preta na cultivar IAC 503 de amendoim, em função dos tratamentos e épocas de avaliação da doença foliar. Pindorama (SP), 2020.

\begin{tabular}{ccc}
\hline Tratamentos. & 90 DAP & 130 DAP \\
\hline T1 & 1,6 & 6,9 \\
T2 & 1,5 & 6,8 \\
T3 & 1,4 & 6,5 \\
T4 & 1,3 & 6,4 \\
T5 & 1,2 & 6,4 \\
T6 & 1,5 & 6,7 \\
T7 & 1,4 & 6,6 \\
\hline $\mathbf{F}$ & 0,91 & 0,98 \\
\hline & CV 2,4\% \\
\hline
\end{tabular}

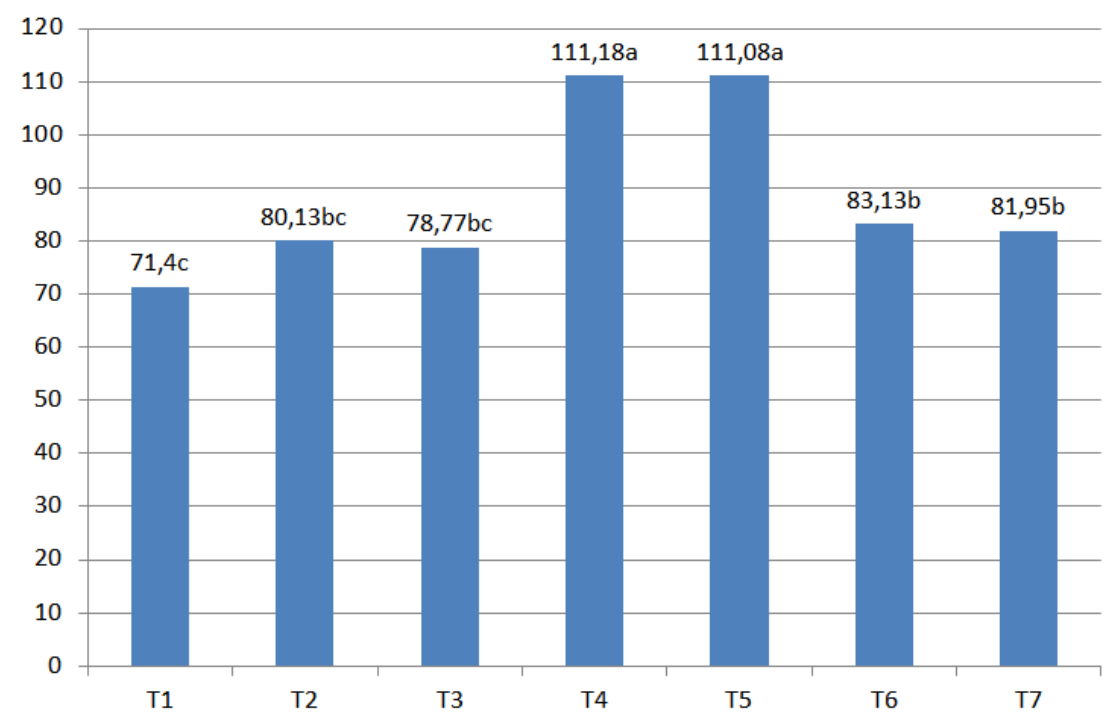

Figura 2. Produtividade (sc.ha-1 ${ }^{-1}$ da cultivar IAC 503 de amendoim, em função dos tratamentos utilizados. Pindorama (SP), 2020.

Conforme mostrado pela Figura 2, os tratamentos que receberam maiores números de aplicações de adubo foliar na ocasião da aplicação de fungicidas foram os que apresentarem maiores produtividades quando comparados aos tratamentos que receberam menos aplicações.

Os tratamentos que receberam adubações foliares apresentaram um acréscimo de produtividade quando comparado ao tratamento padrão regional (T1). Entre os tratamentos o T4 e T5 foram os que obtiveram 
maiores resultados, 35,78 e $35,72 \%$ a mais de produtividade respectivamente.

Como mostra a Tabela 3, quando analisamos isoladamente o T2 e T6 podemos observar que ambos, em uma aplicação, contribuíram com um acrescimento de 11,09 e $14 \%$ na produtividade.

Tabela 3. Relação produtividade entre o cultivo padrão regional (T1) e demais tratamentos. Pindorama, 2020.

\begin{tabular}{crrr}
\hline Relação & Sc.ha $^{-1}$ & $\mathrm{Kg} / \mathrm{ha}^{-1}$ & \multicolumn{1}{c}{$\%$} \\
\hline T2/T1 & 8,73 & 218,00 & 11,09 \\
T3/T1 & 7,37 & 184,50 & 9,35 \\
T4/T1 & 39,78 & 994,00 & 35,78 \\
\hline T5/T1 & 39,68 & 992,00 & 35,68 \\
T6/T1 & 77,73 & 293,25 & 14,00 \\
T7/T1 & 10,50 & 263,75 & 12,88 \\
\hline
\end{tabular}

\section{Conclusões}

Os adubos foliares testados neste ensaio são compatíveis com os fungicidas em mistura de tanque, não prejudicando 0 controle de cercosporioses. A aplicação de adubos foliares proporciona incrementos variáveis na produtividade do amendoim.

\section{Agradecimentos}

Agência Paulista de Tecnologia dos Agronegócios (APTA) - Polo Centro Norte) pela área cedida e implementos utilizados e a Omnia Brasil pelo fornecimento dos produtos Seedboost e Rhizovator. 


\section{Referências}

CONAB. Acompanhamento da Safra Brasileira. 2020. Disponível em: https://www.conab.gov.br/info-agro/safras. Acesso em: 22 jul. 2020.

CRUSCIOL, Carlos Alexandre Costa; SORATTO, Rogério Peres. Nutrição e produtividade do amendoim em sucessão ao cultivo de plantas de cobertura no sistema plantio direto. Pesquisa Agropecuária Brasileira, Brasília, $p$. 1553-1560, nov. 2007. Disponível em: https://ainfo.cnptia.embrapa.br/digital/bitstream/item/106697/1/Nutricao.pdf. Acesso em: 22 jul. 2020.

FOLONI, José Salvador Simoneti et al. EFEITOS DA GESSAGEM E DA ADUBAÇÃO BORATADA SOBRE OS COMPONENTES DE PRODUÇÃO DA CULTURA DO AMENDOIM. Scientia Agraria Paranaensis - Sci. Agrar. Parana., Marechal Cândido Rondon, p. 202-208, jun. 2016. Disponível

em:https://www.alice.cnptia.embrapa.br/alice/bitstream/doc/1062664/1/11419 531571PB.pdf. Acesso em: 22 jul. 2020.

MAPA. BIOINSUMOS. 2020.2 Disponível em: https://www.gov.br/agricultura/pt-br/assuntos/bioinsumos/producaovegetal/fertilidade-de-solo-nutricao-de-plantas-e-tolerancia-a-estressesabioticos. Acesso em: 22 jul. 2020.

SUBRAHMANYAM, P.; MCDONALD, D.; GIBBONS, R. W.; NIGAM, S. N.; NEVILL; D.J.. Resistance to Rust and Late Leafspot Diseases in Some Genotypes of Arachis hypogaea. Peanut Science, [S.I], n. 9, p. 6-10, 1982. Disponível em: https://www.peanutscience.com/doi/10.3146/i0095-3679-9-1$\underline{2}$

SILVA, Ivo Ribeiro da. Matéria orgânica do solo. In: NOVAIS, Roberto Ferreira; VENEGAS, Victor Hugo Alvarez; BARROS, Nairam Félix de; FONTES, Renildes Lúcio Ferreria; CANTARUTTI, Bertola; NEVES, Júlio César Lima. Fertilidade do Solo. Viçosa: Sociedade Brasileira de Ciência do Solo, 2007. p. 3-1017. 